\title{
Identification of CD38 as a potential biomarker in skin cutaneous melanoma using bioinformatics analysis
}

\author{
XIANWANG WANG ${ }^{1,2^{*}}$, PENGLI WANG $^{1 *}$, LEI GE $^{2 *}$, JUAN WANG $^{3}$, \\ SYED MANZAR ABBAS SHAH NAQVI ${ }^{2}$ and SHUJUAN HU ${ }^{4}$
}

\author{
${ }^{1}$ Department of Biochemistry and Molecular Biology, Health Science Center; ${ }^{2}$ Laboratory of Oncology, \\ Center for Molecular Medicine, Health Science Center; ${ }^{3}$ Department of Pediatrics, The Second School of \\ Clinical Medicine and Jingzhou Central Hospital; ${ }^{4}$ Department of Sports Medicine, School of Education and \\ Physical Education, Yangtze University, Jingzhou, Hubei 434023, P.R. China
}

Received November 22, 2019; Accepted June 17, 2020

DOI: $10.3892 / \mathrm{ol} .2020 .11873$

\begin{abstract}
Skin cutaneous melanoma (SKCM) is the most aggressive type of skin cancer, with a high rate of metastasis and mortality; however, identification of biomarkers for the treatment of SKCM is required. Cluster of differentiation (CD)38 has emerged as an effective target for therapeutic drugs in several types of cancer, such as chronic lymphocytic leukemia and multiple myeloma. In the present study, to determine the contribution of CD38 to the diagnosis of SKCM, Gene Expression Profiling Interactive Analysis 2 and University of Alabama Cancer Database online tools were used to analyze The Cancer Genome Atlas-SKCM dataset. Moreover, Search Tool for the Retrieval of Interacting Genes/Proteins and GeneMANIA databases were used to determine protein-protein interaction networks and potential functions. To the best of our knowledge, the results of the present study indicated for the first time that high expression levels of CD38 were a favorable diagnostic factor for SKCM. Moreover, a correlation between CD38 expression levels and the survival probability of patients with SKCM was identified. Integrative analysis predicted that nine genes were correlated with CD38 in SKCM, and the similarity of these genes in SKCM expression and a survival heatmap was verified.
\end{abstract}

Correspondence to: Dr Xianwang Wang, Department of Biochemistry and Molecular Biology, Health Science Center, Yangtze University, 1 Nanhuan Road, Jingzhou, Hubei 434023, P.R. China

E-mail: 275379987@qq.com

Mrs Shujuan $\mathrm{Hu}$, Department of Sports Medicine, School of Education and Physical Education, Yangtze University, 1 Nanhuan Road, Jingzhou, Hubei 434023, P.R. China

E-mail: 523254608@qq.com

${ }^{*}$ Contributed equally

Key words: cluster of differentiation 38, skin cutaneous melanoma, The Cancer Genome Atlas, prognostic biomarker, patient survival
Gene ontology enrichment analysis using the Metascape tool revealed that CD38 and its correlated genes were significantly enriched in lymphocyte activation and $\mathrm{T}$ cell differentiation regulation. Collectively, the bioinformatics analysis revealed that CD38 might serve as a potential diagnostic predictor for SKCM.

\section{Introduction}

Skin cutaneous melanoma (SKCM) is the most aggressive type of skin cancer, with an increasing number of cases worldwide, potential for early metastasis and a high mortality rate (1). The number of treatment options available for human SKCM has increased, including radiotherapy, photodynamic therapy, immunotherapy, chemotherapy and biochemotherapy (2-6). Individualized treatment is difficult to administer to patients with SKCM, as locally advanced SKCM confers a major challenge in terms of surgical and medical management (2). Therefore, effective prognostic markers may provide an alternative therapeutic treatment strategy for patients with SKCM. With the establishment of The Cancer Genome Atlas (TCGA), bioinformatics research has enabled the identification of differentially expressed and mutated genes in different types of human cancer, which has led to the discovery of novel biomarkers from the analysis of TCGA-SKCM samples that display the potential to be used during the diagnosis, treatment and prognosis of SKCM (7-10). However, further investigation into the diagnostic and prognostic biomarkers identified in patients with SKCM is required to determine their suitability, as well as understand the molecular mechanisms and gene networks underlying the development and prognosis of SKCM.

Cluster of differentiation 38 (CD38) is a versatile membrane protein with various functions that was originally identified as a cell surface differentiation marker in B lymphocytes, and was subsequently characterized as a multifunctional enzyme that is expressed ubiquitously (11). Increasing evidence has supported the hypothesis that CD38 is involved during tumorigenesis, tumor growth and metastasis (12-17). In particular, CD38 has been used as a human multiple myeloma target for its combination therapy $(12,15)$. Morandi et al (18) also confirmed that CD38 is expressed in human multiple myeloma 
cells. Furthermore, several studies have revealed that CD38 serves an important role during primary human melanoma metastasis and $\mathrm{T}$ cell proliferation $(13,18)$. The aforementioned studies suggested that CD38 is overexpressed in multiple tumor types, and therefore, may serve as a promising target for therapeutic antibodies and drugs.

To determine the diagnostic and prognostic value of CD38 in patients with SKCM, the publicly available TCGA-SKCM and healthy samples were analyzed using University of Alabama Cancer database (UALCAN) (19) and Gene Expression Profiling Interactive Analysis 2 (GEPIA2) (20) online tools, as well as Search Tool for the Retrieval of Interacting Genes/Proteins (STRING) (21), GeneMANIA (22), cBioPortal for Cancer Genomics (23) and Metascape databases and resources (24). In the present study, the novel diagnostic and prognostic role of CD38 during SKCM was identified. Therefore, the results of the present study might advance the development of antagonist CD38 treatment strategies for patients with SKCM.

\section{Materials and methods}

CD38 expression and mutations analysis. To analyze the expression of CD38 in patients with SKCM, the online software tools UALCAN (ualcan.path.uab.edu/analysis.html) and GEPIA2 (gepia2.cancer-pku.cn/\#index) were used. The UALCAN online tool analyzed CD38 expression levels in SKCM based on sample type, individual cancer stage, and the sex, weight, age and race of the patient. The cBioPortal for Cancer Genomics (www.cbioportal.org) was used to further evaluate the gene expression levels and mutations of CD38 in SKCM samples. All eight TCGA cutaneous melanoma categories were included. All patients with CD38-positive status were included in the present study, and both SKCM and healthy samples were analyzed.

Survival analysis. The Kaplan-Meier method was used to evaluate survival analysis based on the expression of CD38 between various groups. The primary endpoint was disease free survival (DFS), which was defined as the time interval between the initiation of curative treatment and the date of progression, the start date of a second-line treatment or the date of death, whichever occurred first. The secondary endpoint was overall survival (OS), which was defined as the length of time between the date of diagnosis or first therapy to the date of death or last follow-up. The follow-up duration was calculated and presented using the Kaplan-Meier method with 95\% confidence intervals and the log-rank test was used to identify significant differences among the various groups. The effects of CD38 expression levels and the body weight, sex, race of the patient on patient survival were determined using UALCAN. The OS and DFS, based on the expression status of CD38 in patients with SKCM, were determined using GEPIA2. In the total number of patients with SKCM, 50\% displayed high CD38 expression levels $(\mathrm{n}=229 ;>$ median expression) and 50\% displayed low expression levels of CD38 ( $\mathrm{n}=229$; <median expression). Furthermore, the LOGpc database (bioinfo.henu. edu.cn/DatabaseList.jsp) was used to verify the identified OS, platinum-free interval (PFI) and disease-specific survival (DSS) results.
Correlation genes analysis. To identify genes that were correlated with CD38 during SKCM, a correlation analysis was performed. The top 50 genes that displayed a correlation with CD38 expression during SKCM were obtained using the GEPIA2 online tool. The SKCM healthy and tumor expression GDC TCGA Melanoma (SKCM) dataset (dataset ID: TCGA-SKCM.cnv.tsv, http://gepia2.cancer-pku.cn/\#dataset) was analyzed from TCGA (http://gepia2.cancer-pku. $\mathrm{cn} / \#$ similar). Pearson's correlation coefficient (r) was used to screen the top 50 positively correlated genes $(r \geq 0.75)$ with a similar expression pattern to CD38 in SKCM tumor and normal tissues. Furthermore, the UALCAN tool was used to determine the correlation between nine highly correlated genes and CD38.

Expression and survival heatmap analysis. The heatmap profile of nine correlated genes with CD38 expression during SKCM and 32 additional TCGA cancer types, including adrenocortical carcinoma, bladder urothelial carcinoma, breast invasive carcinoma, cervical squamous cell carcinoma and endocervical adenocarcinoma, cholangiocarcinoma, colon adenocarcinoma, lymphoid neoplasm diffuse large B cell lymphoma, esophageal carcinoma, glioblastoma multiforme, head and neck squamous cell carcinoma, kidney chromophobe, kidney renal clear cell carcinoma, kidney renal papillary cell carcinoma, acute myeloid leukemia, brain lower grade glioma, liver hepatocellular carcinoma, lung adenocarcinoma, lung squamous cell carcinoma, mesothelioma, ovarian serous cystadenocarcinoma, pancreatic adenocarcinoma, pheochromocytoma and paraganglioma, prostate adenocarcinoma, rectum adenocarcinoma, sarcoma, stomach adenocarcinoma, testicular germ cell tumors, thyroid carcinoma, thymoma, uterine corpus endometrial carcinoma, uterine carcinosarcoma, uveal melanoma, were generated using an interactive heatmap and the multiple gene comparison tools in GEPIA2. Both healthy and tumor data from TCGA were selected for the expression heatmap analysis. To compare the survival contribution of the top nine correlated genes with CD38 expression during SKCM and additional TCGA cancer types, the survival map was calculated from TCGA-tumor specimens using the Mantel-Cox test.

Occurrence of CD38 isoforms and promoter methylation analysis. To verify the five structural isoforms of CD38 and to compare the occurrence in SKCM, the TCGA-SKCM dataset in the GEPIA2 online tool was used with the following parameters: Cancer, $\mathrm{X}$ and isoform, Y. To analyze the CD38 promoter methylation levels in patients with SKCM, the CD38 promoter methylation profile based on individual cancer stage, and the sex, weight and age of the patient was analyzed using the UALCAN online tool.

Protein-protein interaction (PPI) networks and Gene Ontology (GO) enrichment analysis. The STRING database (string-db.org) provides a critical evaluation and integration of PPI, including physical and functional relevance (21). The PPI network of CD38 was produced using STRING online tools (version 10.0). GeneMANIA (genemania.org) is a flexible user-friendly website for generating hypotheses regarding gene function, analyzing gene lists and prioritizing 
A
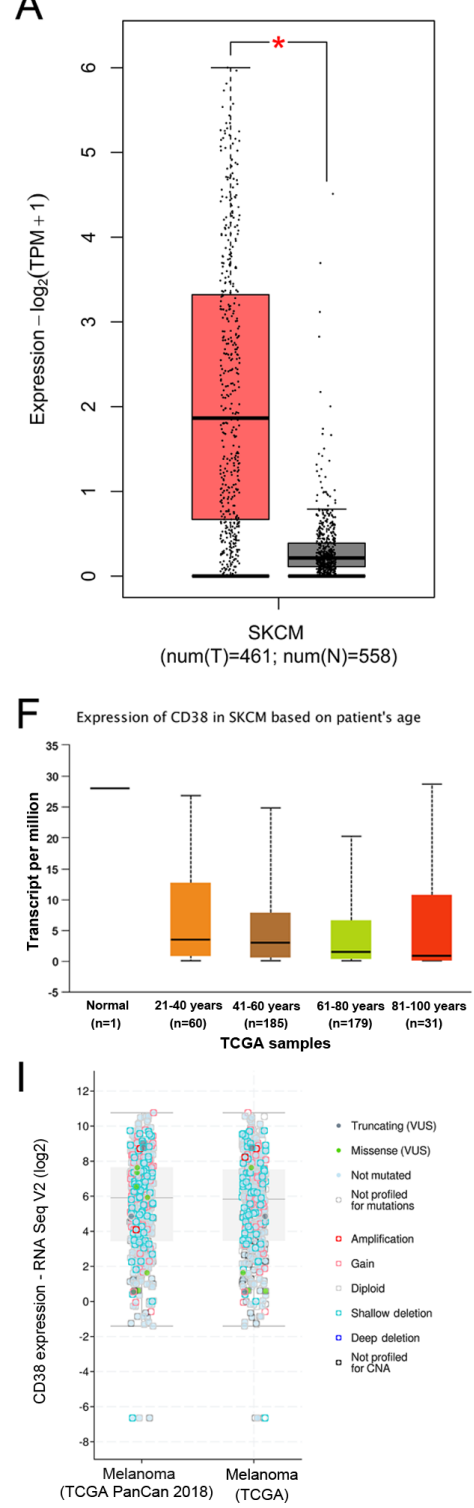

B

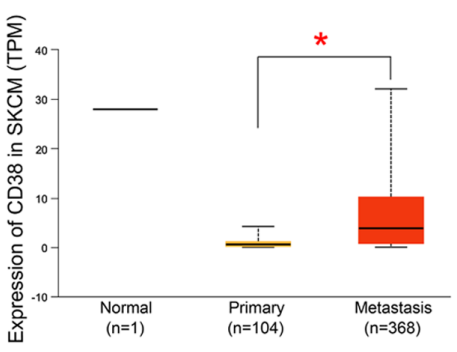

D Expression of $\mathrm{CD} 38$ in SKCM based on patient's weight

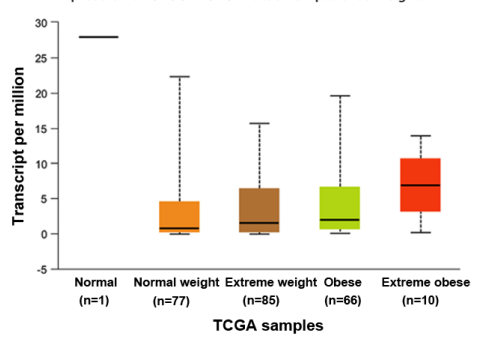

G

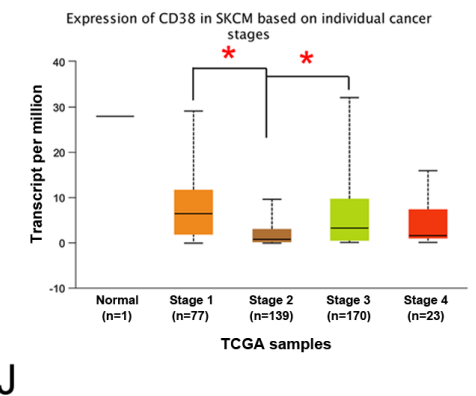

C

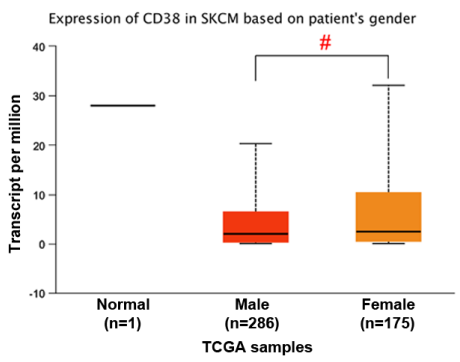

E

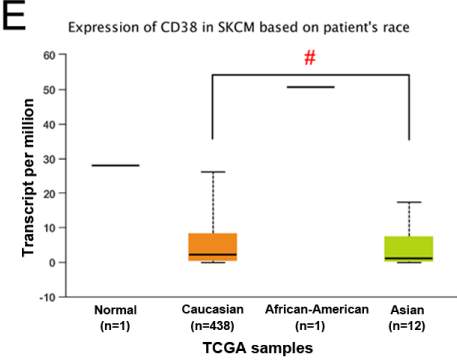

$\mathrm{H}$

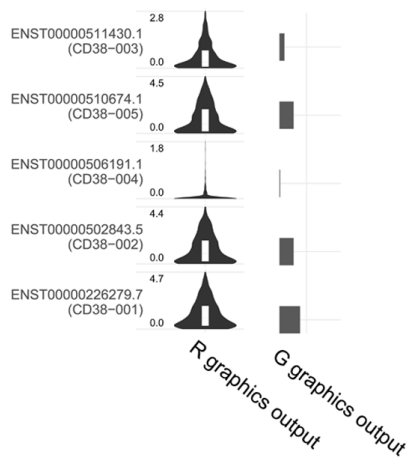

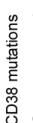

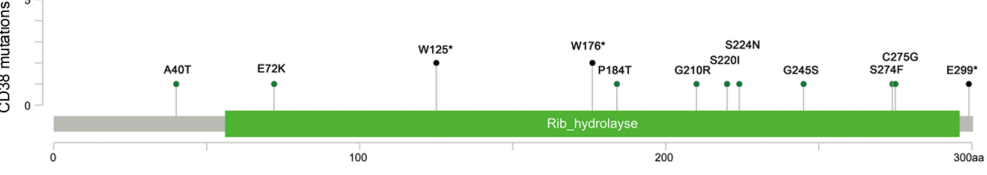

Figure 1. CD38 expression and mutations in patients with SKCM. (A) CD38 expression in SKCM using (A) the GEPIA2 online tool and (B) UALCAN. CD38 expression in SKCM based on (C) sex, (D) body weight, (E) race, (F) age and (G) individual cancer stage of the patient. (H) Occurrence of CD38 isoforms in patients from TGGA-SKCM dataset (X, cancer; Y, isoform). (I) CD38 expression and mutations analyzed using cBioPortal for Cancer Genomic. (J) Location of specific CD38 mutations in patients with SKCM. Green and black represent missense and truncating mutations, respectively. ${ }^{*} \mathrm{P}<0.05 ;{ }^{*} \mathrm{P}<0.001 . \mathrm{CD} 38$, cluster of differentiation 38; SKCM, skin cutaneous melanoma; GEPIA2, Gene Expression Profiling Interactive Analysis 2; UALCAN, University of Alabama Cancer database; TCGA, The Cancer Genome Atlas; TPM, Transcripts Per Million; T, tumor; N, normal.

genes for functional assays. The Metascape tool (metascape. org) provides a resource for biologists for the analysis of systems-level datasets (24). Therefore, GeneMANIA and Metascape were used to further analyze the related genes and functional enrichment of CD38.

\section{Results}

Elevated expression level and specific mutations of CD38 in patients with SKCM from TCGA dataset. To determine the expression level of CD38 in patients with SKCM, the online analytical UALCAN and GEPIA2 tools were used. The expression level of CD38 was significantly higher in SKCM tissues $(n=461)$ compared with healthy tissues $(n=558 ; P<0.001$; Fig. 1A). From the UALCAN analysis, CD38 expression levels were significantly increased in SKCM metastatic tumors compared with primary tumors ( $\mathrm{P}<0.001 ;$ Fig. 1B). In addition, there was a significant difference in CD38 expression levels between the sex $(\mathrm{P}<0.05$; Fig. $1 \mathrm{C})$, race $(\mathrm{P}<0.05$; Caucasian vs. Asian; Fig. 1E) and individual cancer stage $(\mathrm{P}<0.001$; stage 1 vs. stage 2 and stage 2 vs. stage 3; Fig. 1G) of the patient, but there were no significant differences in the expression levels of CD38 between the weight (Fig. 1D) and age (Fig. 1F) of the patient.

In addition, the GEPIA2 analysis indicated that the occurrence of the two major isoforms, CD38-001 and CD38-005, are more frequent in patients with SKCM compared with other isoforms (Fig. 1H). The cBioPortal for Cancer Genomics analysis suggested that there were a total of 22 mutations in CD38 in TCGA-SKCM samples, including 14 missenses and 
Table I. Mutations in CD38 gene in TCGA-SKCM dataset.

\begin{tabular}{llllcrr}
\hline Sample ID & Protein change & $\begin{array}{c}\text { Mutation } \\
\text { type }\end{array}$ & $\begin{array}{c}\text { Copy } \\
\text { vamber } \\
\text { variation }\end{array}$ & $\begin{array}{c}\text { Number of } \\
\text { occurrences in } \\
\text { COSMIC }\end{array}$ & $\begin{array}{c}\text { Allele } \\
\text { frequency } \\
\text { (total) }\end{array}$ & $\begin{array}{r}\text { Number of } \\
\text { mutations } \\
\text { in sample }\end{array}$ \\
\hline MEL-JWCI-WGS-12 & C275G & Missense & NA & NA & NA & 2703 \\
TCGA-FS-A1ZK-06 & W176 & Nonsense & Gain & 2 & 0.16 & 1297 \\
TCGA-EE-A2MR-06 & S274F & Missense & Diploid & 1 & 0.14 & 3944 \\
TCGA-FW-A3R5-06 & W125a & Nonsense & Diploid & 1 & 0.18 & 15939 \\
TCGA-GF-A6C9-06 & W125 & Nonsense & ShallowDel & 1 & 0.18 & 2762 \\
TCGA-EB-A5UN-06 & G245S & Missense & Diploid & 1 & 0.19 & 625 \\
TCGA-FW-A3R5-06 & E72K & Missense & Diploid & 1 & 0.37 & 15939 \\
TCGA-BF-A5ER-01 & A40T & Missense & NA & NA & 0.38 & 165 \\
TCGA-EB-A6QY-01 & S224N & Missense & NA & NA & 0.24 & 518 \\
TCGA-FS-A1ZK-06 & W176 & Nonsense & Gain & 2 & 0.16 & 1459 \\
TCGA-EE-A2MR-06 & S274F & Missense & Diploid & 1 & 0.14 & 2921 \\
TCGA-FW-A3R5-06 & W125 & Nonsense & Diploid & 1 & 0.17 & 15832 \\
TCGA-GF-A6C9-06 & W125 & Nonsense & ShallowDel & 1 & 0.15 & 2318 \\
TCGA-EB-A5UN-06 & G245S & Missense & Diploid & 1 & 0.19 & 627 \\
TCGA-FW-A3R5-06 & E72K & Missense & Diploid & 1 & 0.38 & 15832 \\
TCGA-BF-A5ER-01 & A40T & Missense & NA & NA & 0.37 & 159 \\
TCGA-EB-A6QY-01 & S224N & Missense & NA & NA & 0.24 & 506 \\
TCGA-D3-A2JL-06 & P184T & Missense & Diploid & NA & 0.11 & 1202 \\
TCGA-EE-A183-06 & S220I & Missense & Diploid & 1 & 0.12 & 2156 \\
TCGA-FS-A1Z4-06 & E299 & Nonsense & ShallowDel & NA & 0.1 & 609 \\
TCGA-Z2-A8RT-06 & G210R & Missense & ShallowDel & NA & 0.68 & 2422 \\
YUKLAB & W176a & Nonsense & NA & 2 & NA & 3656 \\
\hline
\end{tabular}

${ }^{a}$ Termination codon; CD, cluster of differentiation; TCGA, The Cancer Genome Atlas; SKCM, skin cutaneous melanoma; COSMIC, Catalogue of Somatic Mutations in Cancer; NA, not available; Diploid, normal; ShallowDel, Shallow deletion; Gain, Low-level gain.

8 truncating mutations (Fig. 1I and J; Table I). Among them, the W125 mutation affects CD38 enzyme activity, and the single nucleotide polymorphism (SNP) at position (P184) is associated with autism spectrum disorder and type II diabetes, and C275 is a disulfide bond formation site for CD38 (11). Collectively, the results from the present study suggested that high expression levels and key mutations in CD38 might be involved in the development of SKCM.

Effect of CD38 expression level on patient survival in TCGA-SKCM dataset. To evaluate the prognostic value of CD38 in patients with SKCM, the effect of CD38 expression levels on survival time was analyzed. Higher CD38 expression levels resulted in a significantly higher survival probability compared with lower CD38 expression levels $(\mathrm{P}<0.0001$; Fig. 2A). In addition, survival probability was also associated with the body weight, sex and race of the patient. Low/medium CD38 expression levels in Asian patients resulted in a shorter survival time compared with patients from other ethnicities $(\mathrm{P}<0.0001$; Fig. 2B). CD38 expression levels and the sex $(\mathrm{P}=0.00029)$ and body weight $(\mathrm{P}=0.021)$ of the patients were also significantly associated with the survival of patients with SKCM (Fig. 2C and D). In particular, female patients diagnosed with SKCM displayed a longer survival time compared with male patients.
Moreover, the OS and DFS of CD38 expression levels in patients with SKCM were analyzed using the GEPIA2 online tool. Patients with high CD38 expression levels displayed a higher survival probability for both OS and DFS compared with patients with low CD38 expression levels (Fig. 2E and F). The contribution of the CD38 isoforms to the survival of patients with SKCM was determined. The survival map analysis verified that major isoform, CD38-001, affected the survival of patients with SKCM (Fig. 2G and H).

To verify the aforementioned results, the LOGpc database was used. Patients with high CD38 expression levels displayed higher OS $(\mathrm{P}<0.0001)$, PFI $(\mathrm{P}<0.0001)$ and DSS $(\mathrm{P}<0.005)$ probabilities compared with patients with low CD38 expression levels (Fig. 2I-K).

Correlated genes with CD38 in patients with SKCM from TCGA dataset. To identify genes correlated with CD38 in SKCM, a correlation analysis was conducted. The top 50 correlated genes with CD38 expression in SKCM were investigated using GEPIA2 (Table II; Pearson $\mathrm{CC} \geq 0.75$ ). A PPI network of these genes was created using the STRING tool (Fig. 3A). The identified genes were from diverse functional groups, such as cell adhesion molecules (CD80, CD86, CD8A, CD8B, CD2, ICOS, ITGAL, ITGB7 and TIGIT), chemokine signaling pathway molecules (CCL4, CCL25, 

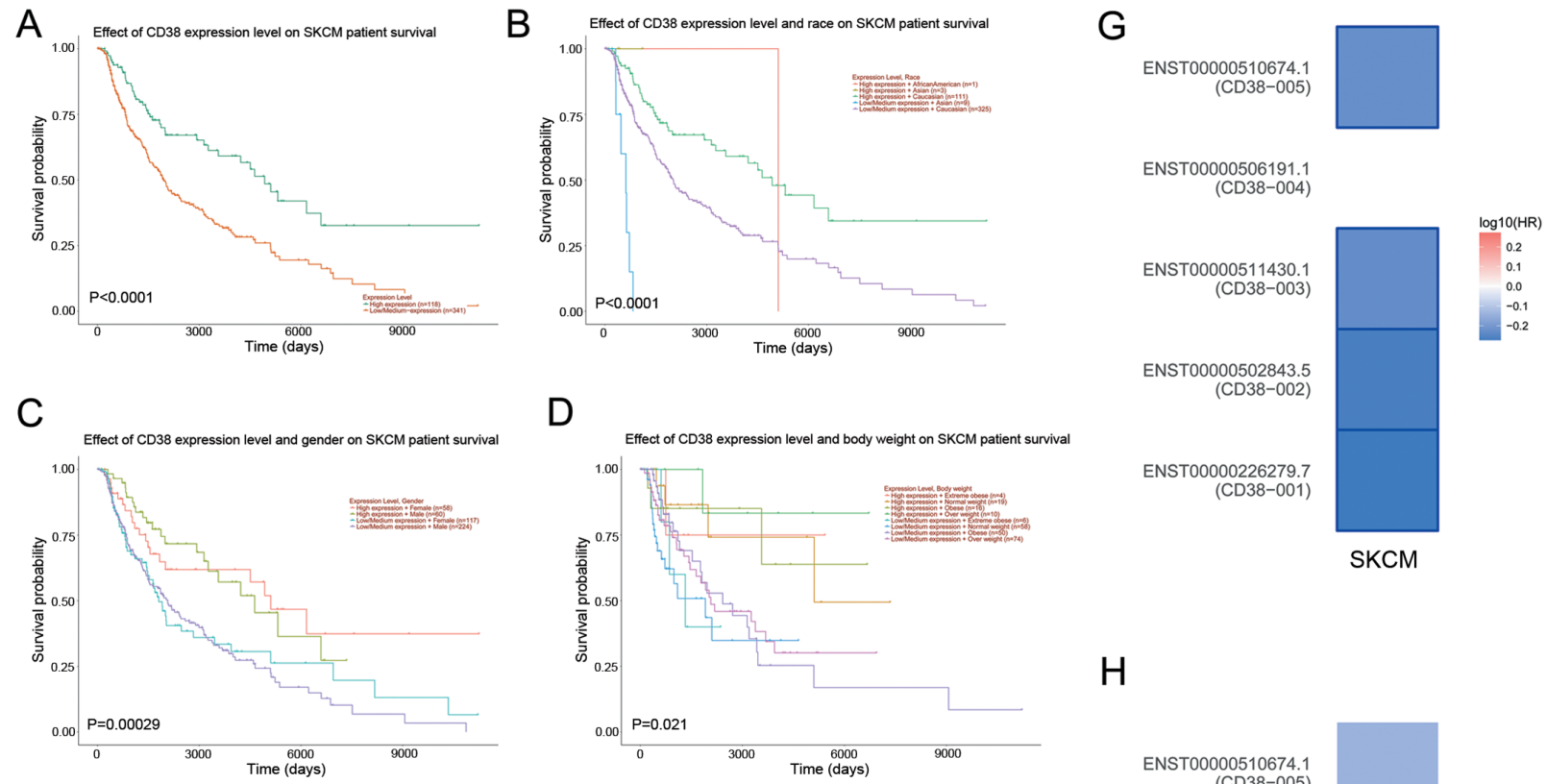

E

F
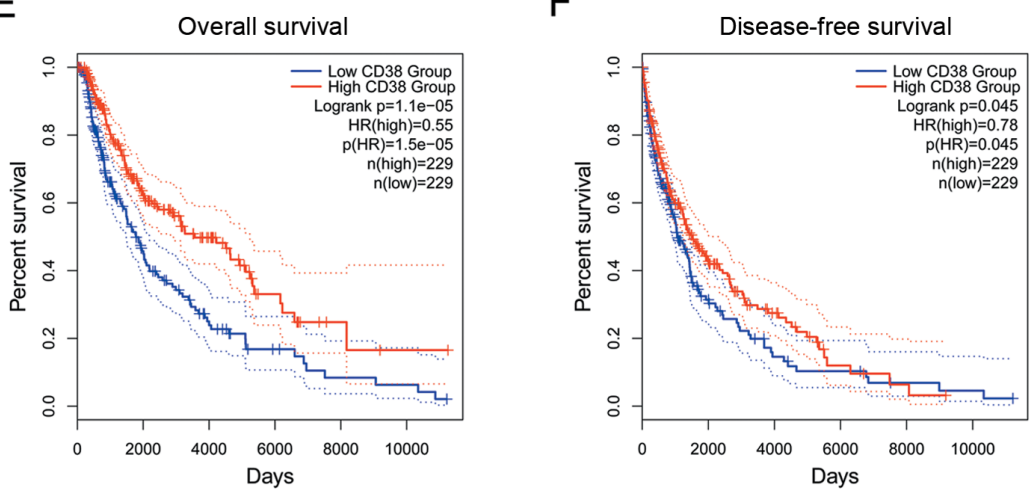

$\mathrm{H}$
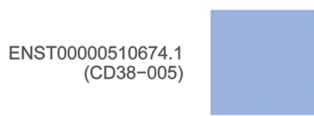

ENSTO00000506191.1
(CD38-004)

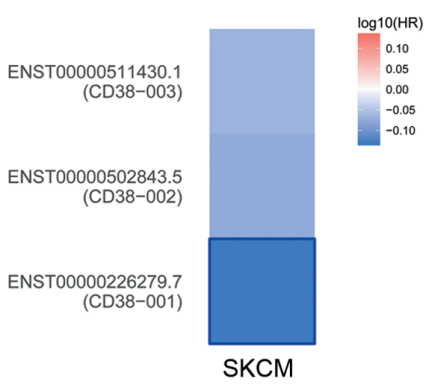

I
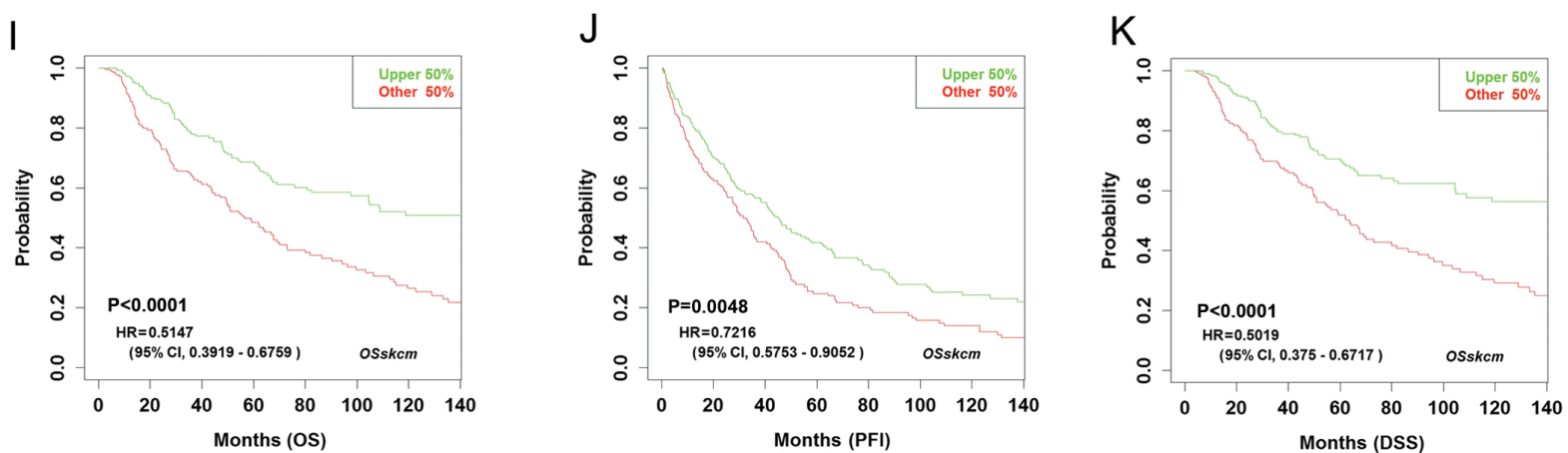

Figure 2. Effect of CD38 expression on overall survival in patients with SKCM. (A) Higher CD38 expression was associated with improved survival probability in patients with SKCM. Effect of CD38 expression and (B) race, (C) sex and (D) body weight on survival in patients with SKCM. (E) OS and (F) DFS were identified using the GEPIA2 online tool. (G) OS and (H) DFS heatmaps of five CD38 isoforms in patients with SKCM. (I) OS, (J) PFI and (K) DSS analysis using the LOGpc Database. CD38, cluster of differentiation 38; SKCM, skin cutaneous melanoma; OS, overall survival; DFS, disease-free survival; GEPIA2, Gene Expression Profiling Interactive Analysis 2; PFI, platinum-free interval; DSS, disease-specific survival; CI, confidence interval; HR, hazard ratio.

CXCR6, CXCL9, CCR2 and GNGT2) and intestinal immune network for IgA production molecules (CD80, CD86, ICOS, ITGB7 and CCL25). A total of nine correlated genes that interacted with CD38 were further predicted using the STRING database (Fig. 3B), which included CD86, CD80, CD27, CD2, IL21R, IL2RB, ITGAL, CCL4 and TBX21. Functional enrichment analysis from Metascape revealed that the nine correlated genes with CD38 in patients with
SKCM might be involved with lymphocyte activation and regulation of $\mathrm{T}$ cell differentiation (Fig. 3C).

In addition, the correlation between CD38 and the nine aforementioned correlated genes was further illustrated in Fig. 4A. To determine the similarity of the correlated genes, expression and survival heatmaps across TCGA tumors were generated. The survival heatmaps of the nine aforementioned CD38-correlated genes displayed a positive correlation with 
Table II. Top 50 ranked CD38 correlated genes in TCGA-SKCM dataset.

\begin{tabular}{|c|c|c|c|}
\hline Genenumber & Gene symbol & Gene ID & $\mathrm{r}$ \\
\hline 1 & TIGIT & ENSG00000181847.11 & 0.82 \\
\hline 2 & TBX21 & ENSG00000073861.2 & 0.81 \\
\hline 3 & PLEK & ENSG00000115956.9 & 0.81 \\
\hline 4 & ZBP1 & ENSG00000124256.14 & 0.81 \\
\hline 5 & CCR2 & ENSG00000121807.5 & 0.81 \\
\hline 6 & LAX1 & ENSG00000122188.12 & 0.80 \\
\hline 7 & SLA & ENSG00000155926.13 & 0.80 \\
\hline 8 & SAMSN1 & ENSG00000155307.17 & 0.80 \\
\hline 9 & CXCR6 & ENSG00000172215.5 & 0.79 \\
\hline 10 & PIM2 & ENSG00000102096.9 & 0.79 \\
\hline 11 & SIRPG & ENSG00000089012.14 & 0.79 \\
\hline 12 & GBP5 & ENSG00000154451.14 & 0.78 \\
\hline 13 & GNGT2 & ENSG00000167083.6 & 0.78 \\
\hline 14 & SLA2 & ENSG00000101082.13 & 0.78 \\
\hline 15 & SLAMF6 & ENSG00000162739.13 & 0.78 \\
\hline 16 & IL2RB & ENSG00000100385.13 & 0.78 \\
\hline 17 & SH2D1A & ENSG00000183918.14 & 0.78 \\
\hline 18 & AKAP5 & ENSG00000179841.8 & 0.77 \\
\hline 19 & $\mathrm{GCH} 1$ & ENSG00000131979.18 & 0.77 \\
\hline 20 & ITGAL & ENSG00000005844.17 & 0.77 \\
\hline 21 & $\mathrm{CD} 2$ & ENSG00000116824.4 & 0.77 \\
\hline 22 & SCIMP & ENSG00000161929.14 & 0.76 \\
\hline 23 & USP30-AS1 & ENSG00000256262.1 & 0.76 \\
\hline 24 & ICOS & ENSG00000163600.12 & 0.76 \\
\hline 25 & IL10RA & ENSG00000110324.9 & 0.76 \\
\hline 26 & CD86 & ENSG00000114013.15 & 0.76 \\
\hline 27 & IL21R & ENSG00000103522.15 & 0.76 \\
\hline 28 & GIMAP5 & ENSG00000196329.10 & 0.76 \\
\hline 29 & GIMAP4 & ENSG00000133574.9 & 0.76 \\
\hline 30 & IGSF6 & ENSG00000140749.8 & 0.76 \\
\hline 31 & NCKAP1L & ENSG00000123338.12 & 0.76 \\
\hline 32 & TRGV10 & ENSG00000211694.2 & 0.76 \\
\hline 33 & CD8B & ENSG00000172116.21 & 0.76 \\
\hline 34 & CXCL9 & ENSG00000138755.5 & 0.76 \\
\hline 35 & CXCR2P1 & ENSG00000229754.1 & 0.76 \\
\hline 36 & TRGC2 & ENSG00000227191.6 & 0.76 \\
\hline 37 & FAM26F & ENSG00000188820.12 & 0.75 \\
\hline 38 & ITGB7 & ENSG00000139626.15 & 0.75 \\
\hline 39 & GPR171 & ENSG00000174946.6 & 0.75 \\
\hline 40 & CD8A & ENSG00000153563.15 & 0.75 \\
\hline 41 & TRGV2 & ENSG00000233306.2 & 0.75 \\
\hline 42 & CD80 & ENSG00000121594.11 & 0.75 \\
\hline 43 & ABCD2 & ENSG00000173208.3 & 0.75 \\
\hline 44 & CD27 & ENSG00000139193.3 & 0.75 \\
\hline 45 & RP11-493L12.5 & ENSG00000257924.1 & 0.75 \\
\hline 46 & DOK2 & ENSG00000147443.12 & 0.75 \\
\hline 47 & LILRB1 & ENSG00000104972.14 & 0.75 \\
\hline 48 & CCL4 & ENSG00000275302.1 & 0.75 \\
\hline 49 & CCL25 & ENSG00000131142.13 & 0.75 \\
\hline 50 & AC104820.2 & ENSG00000234663.5 & 0.75 \\
\hline
\end{tabular}

CD, cluster of differentiation; TCGA, The Cancer Genome Atlas; SKCM, skin cutaneous melanoma. 


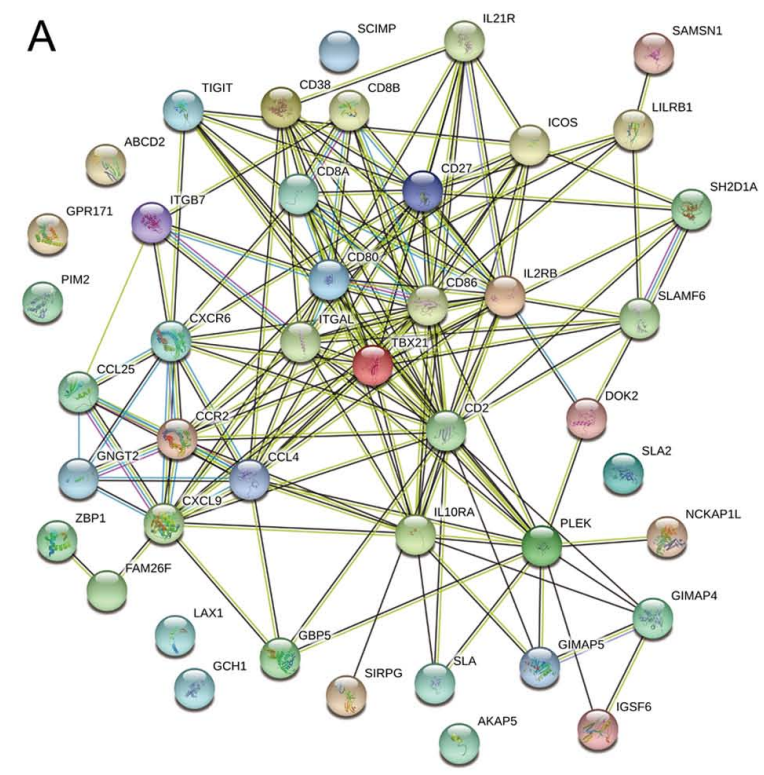

B

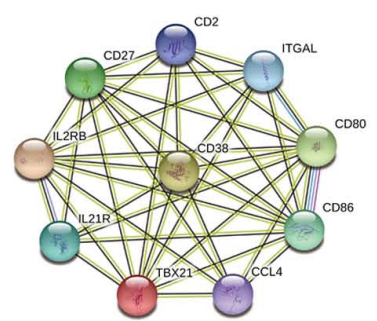

C

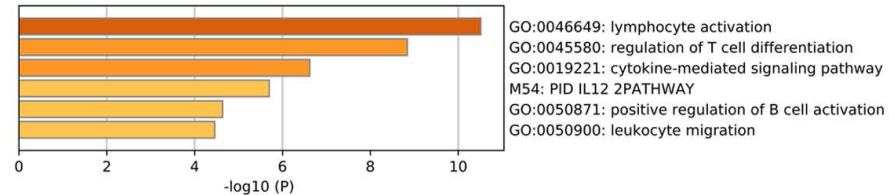

Figure 3. CD38-correlated genes and potential functions in patients with SKCM. Protein-protein interaction network of CD38 with (A) the top 50 ranked correlated genes and (B) nine correlated genes in SKCM. (C) Gene Ontology enrichment analysis of six potential functional processes of the nine correlated genes in SKCM. CD38, cluster of differentiation 38; SKCM, skin cutaneous melanoma.

survival in patients with SKCM, which was consistent with the results of CD38, but there was no correlation with other types of tumor. Moreover, the expression heatmaps in patients with SKCM and healthy tissues of the nine aforementioned genes was similar to the expression heatmap of CD38 (Fig. 4C). However, further studies are required to validate the correlated genes and to explore underlying functional mechanisms.

Promoter methylation levels of CD38 in patients with SKCM from TCGA dataset. To investigate the CD38 promoter methylation profile in patients with SKCM based on the cancer type, individual cancer stage, age, sex, race and weight of the patient, the UALCAN online tool was used. The results suggested that the individual cancer stage $(\mathrm{P}<0.05$; stage 2 vs. stage 3; Fig. 5B), age ( $\mathrm{P}<0.05$; 41-60 vs. 21-40/61-80 years; Fig. 5C) and sex $(\mathrm{P}<0.05$; male vs. female; Fig. 5D) of the patient might contribute to the promoter methylation level of CD38 in patients with SKCM. However, there were no significant alterations in the promoter methylation levels of CD38 in the TCGA-SKCM dataset based on the cancer type (Fig. 5A), race (Fig. 5E) or weight (Fig. 5F) of the patient.

PPI network and GO enrichment analysis of CD38. The functional interactions between proteins can provide important information of the molecular mechanism involved. The PPI network of CD38 was determined using the STRING database (Fig. 6A). The results indicated that CD38 interacted with 10 functional genes, including NMNAT1, NMNAT2, NMNAT3, NAMPT, ENPP1, ENPP3, NADK, BST1, PNP and NNMT. From the STRING functional enrichments, the 10 associated genes were involved in the nucleotide metabolic process and nine of the genes were involved in the nicotinamide adenine dinucleotide (NAD) metabolic process. A further 20 genes interacting with CD38 were identified using GeneMANIA (Fig. 6B). Among them, LCK, CD3E, CD247 CD4 and FCGR3A were identified by physical interactions with CD38.
In particular, the functional enrichment analysis revealed that the proteins were involved in the immune response activating cell surface receptor signaling pathway. RYR1, RYR2 and RYR3 were also implicated in calcium release channel activity and intracellular ligand-gated calcium channel activity process. Consistent with the aforementioned correlation analysis, ITGAL belonged to the group of nine correlated genes with CD38 in patients with SKCM.

To further determine the potential function of CD38 in SKCM, GO enrichment analysis of CD38 and the genes it interacts with was performed using Metascape. The results suggested that CD38 and the 10 genes it interacted with were enriched in nicotinate and nicotinamide metabolism, metabolism of water-soluble vitamins and cofactors, and nicotinate metabolism processes $\left(-\log _{10}(\mathrm{P})>20\right.$; Fig. 6C). A total of 20 CD38-correlated genes identified using GeneMANIA were significantly enriched in translocation of ZAP-70 to immunological synapse, cellular response to caffeine, immunoregulatory interactions between a lymphoid and a non-lymphoid cell, and natural killer cell mediated cytotoxicity $\left(-\log _{10}(\mathrm{P})>6\right.$; Fig. $\left.6 \mathrm{D}\right)$.

\section{Discussion}

Over the past few years, several reports have concentrated on differentially expressed genes and mutations in patients with SKCM, identifying diagnostic and prognostic biomarkers for SKCM (8,25-27). However, the current curative biomarkers identified for the therapy of patients with SKCM are not adequate (28). CD38 has emerged as an effective target for therapeutic antibodies and drugs in human multiple myeloma and neuroblastoma $(14-16,29)$. The present study indicated that higher CD38 expression levels and specific CD38 mutations were favorable diagnostic factors in SKCM. Both UALCAN and GEPIA2 online tools revealed that the expression level of CD38 was significantly higher in patients with SKCM 

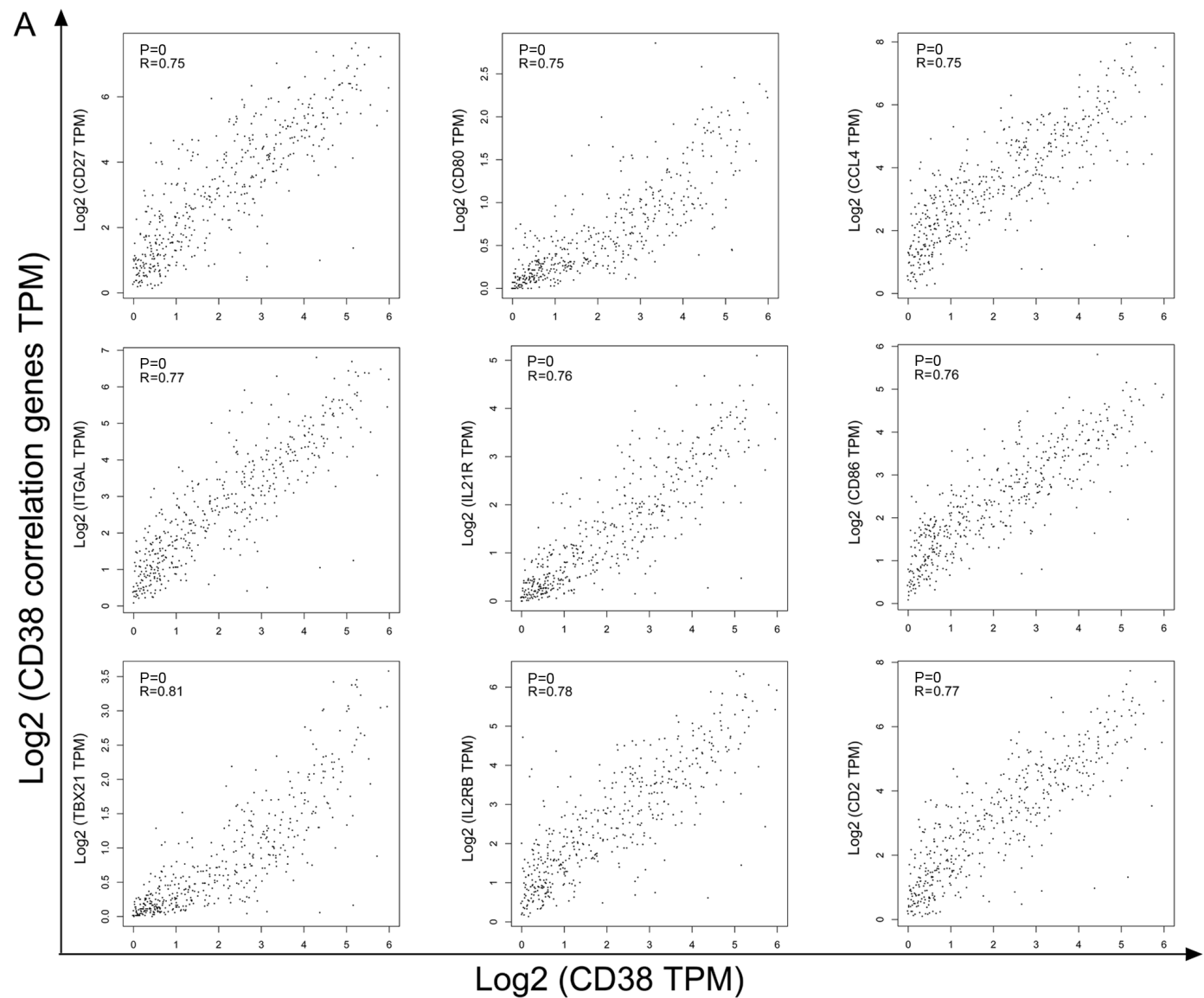

B

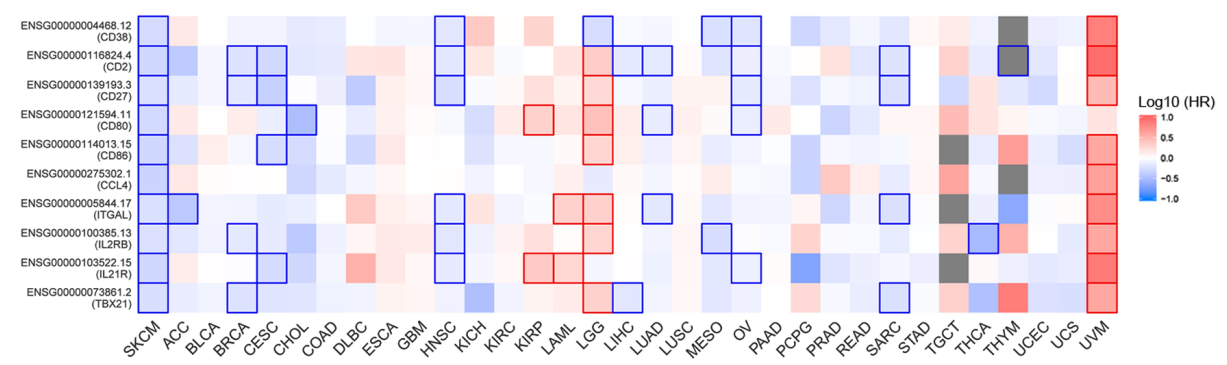

C

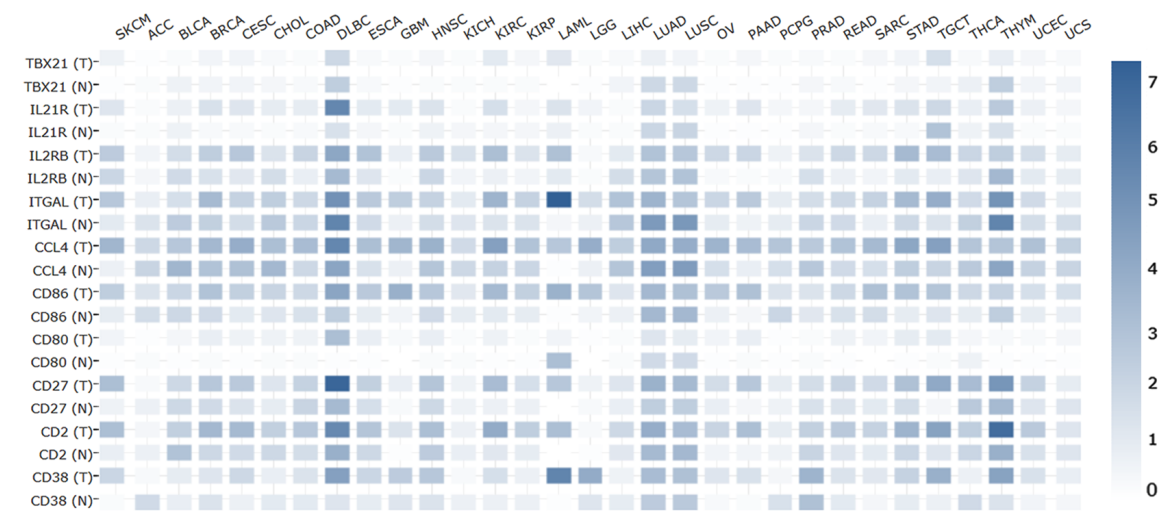

Figure 4. Correlation between CD38 and nine correlated genes. (A) Correlation analysis of CD86, CD80, CD27, CD2, IL21R, IL2RB, ITGAL, CCL4 and TBX21 with CD38 in patients with SKCM. (B) Survival heatmap of nine correlated genes and CD38 in SKCM and other TCGA tumors. (C) Expression heatmap of nine correlated genes and CD38 across TCGA tumors. The shades of blue represent the expression levels of these genes in different tissues (normal and tumor). $\log _{2}(\mathrm{TPM}+1)$ transformed expression data were chosen for plotting. CD38, cluster of differentiation 38; SKCM, skin cutaneous melanoma; ACC, adrenocortical carcinoma; BLCA, bladder urothelial carcinoma; BRCA, breast invasive carcinoma; CESC, cervical squamous cell carcinoma and endocervical adenocarcinoma; CHOL, cholangiocarcinoma; COAD, colon adenocarcinoma; DLBC, lymphoid neoplasm diffuse large B cell lymphoma; ESCA, esophageal carcinoma; GBM, glioblastoma multiforme; HNSC, head and neck squamous cell carcinoma; KICH, kidney chromophobe; KIRC, kidney renal clear cell carcinoma; KIRP, kidney renal papillary cell carcinoma; LAML, acute myeloid leukemia; LGG, brain lower grade glioma; LIHC, liver hepatocellular carcinoma; LUAD, lung adenocarcinoma; LUSC, lung squamous cell carcinoma; MESO, mesothelioma; OV, ovarian serous cystadenocarcinoma; PAAD, pancreatic adenocarcinoma; PCPG, pheochromocytoma and paraganglioma; PRAD, prostate adenocarcinoma; READ, rectum adenocarcinoma; SARC, sarcoma; STAD, stomach adenocarcinoma; TGCT, testicular germ cell tumors; THCA, thyroid carcinoma; THYM, thymoma; UCEC, uterine corpus endometrial carcinoma; UCS, uterine carcinosarcoma; UVM, uveal melanoma; TCGA, The Cancer Genome Atlas; TPM, Transcripts Per Million; HR, hazard ratio. 
A Promoter methylation level of CD38 in SKCM

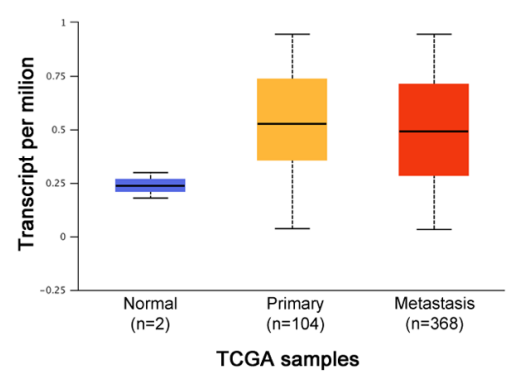

D

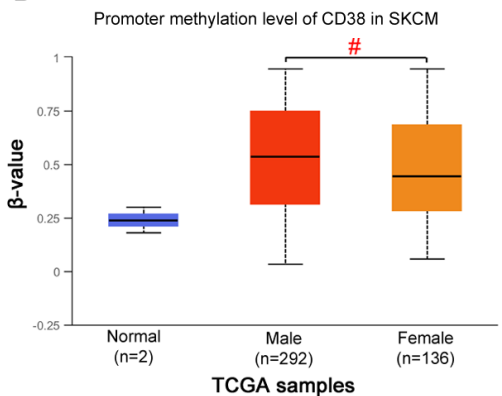

B

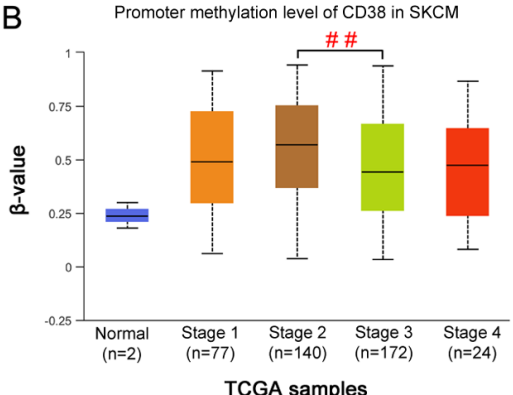

E

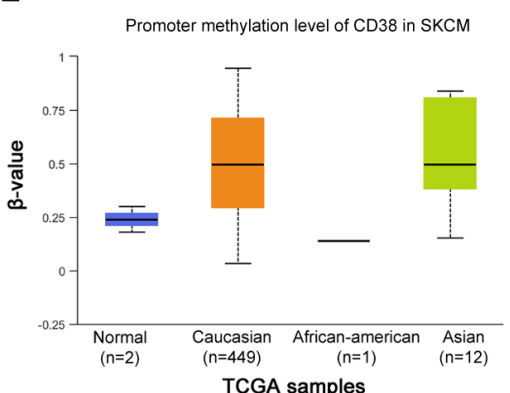

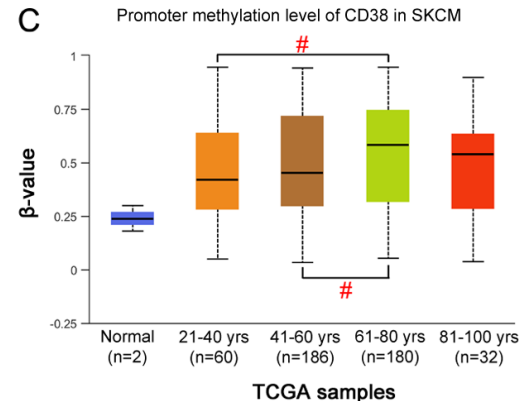

$\mathrm{F}$

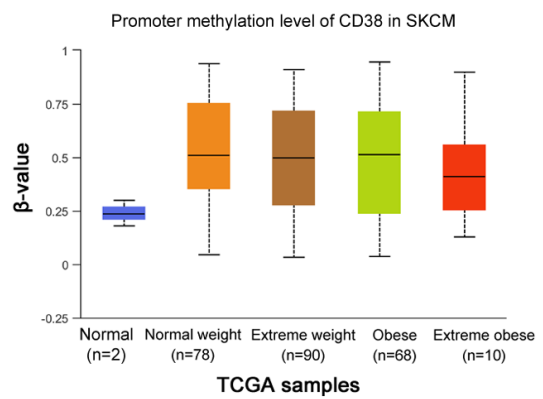

Figure 5. Promoter methylation levels of CD38 in patients with SKCM. Promoter methylation levels of CD38 in patients with SKCM based on the (A) cancer type, (B) individual cancer stages, (C) age, (D) sex, (E) race and (F) body weight of the patient. ${ }^{\#} \mathrm{P}<0.05$ and ${ }^{\# \#} \mathrm{P}<0.005$. CD38, cluster of differentiation 38; SKCM, skin cutaneous melanoma; TCGA, The Cancer Genome Atlas.

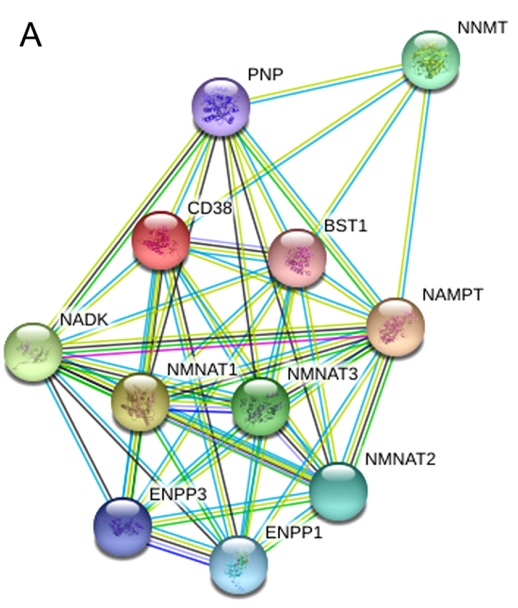

C

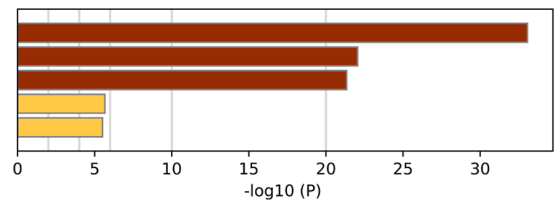

hsa00760: Nicotinate and nicotinamide metabolism R-HSA-196849: Metabolism of water-soluble vitamins and cofactors R-HSA-196807: Nicotinate metabolism GO:0070663: regulation of leukocyte proliferation GO:0006163: purine nucleotide metabolic process

D

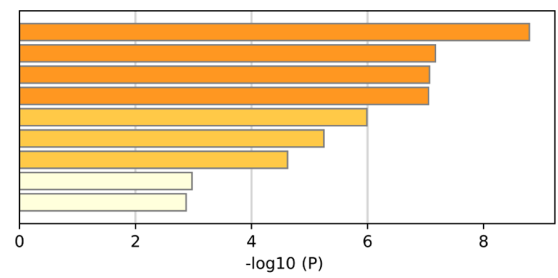

R-HSA-202430: Translocation of ZAP-70 to Immunological synapse GO:0071313: cellular response to caffeine R-HSA-198933: Immunoregulatory interactions between a Lymphoid and a non-Lymphoid cell hsa04650: Natural killer cell mediated cytotoxicity hsa04970: Salivary secretion

R-HSA-202733: Cell surface interactions at the vascular wall

M272: PID CD8 TCR DOWNSTREAM PATHWAY GO:0098542: defense response to other organism

Figure 6. PPI networks and GO enrichment analysis of CD38. (A) PPI network of CD38 and its interactive genes. (B) PPI network of CD38 generated using GeneMANIA. (C) GO enrichment analysis of CD38 and the top 10 interactive genes obtained from the PPI network of CD38 and its interactive genes. (D) GO enrichment analysis of CD38 and the top 20 interactive genes obtained from the PPI network of CD38 generated using GeneMANIA. PPI, protein-protein interaction; GO, Gene Ontology; CD38, cluster of differentiation 38. 
compared with healthy tissues. Furthermore, the results indicated that high expression of CD38 may serve as a biomarker for SKCM metastasis. In addition, several specific mutations of CD38 were identified in patients with SKCM. In particular, $\mathrm{W} 125$ is one of the key residues, which is responsible for nucleosidase activity of CD38 (30), P184 is a common SNP (11), and the cysteine residues between $\mathrm{C} 275$ and $\mathrm{C} 254$ form a disulfide bond within CD38 (11). The aforementioned mutations or polymorphisms might increase the risk of developing SKCM. In addition, a positive correlation between CD38 expression levels and survival probability in patients with SKCM was identified. The results suggested that higher CD38 expression levels resulted in improved survival probability, which could affect the sensitization of chemotherapy drugs or antibodies.

To the best of our knowledge, the present study also screened the top 50 correlated genes with CD38 in patients with SKCM for the first time, which might be implicated in the prognosis of SKCM. However, further studies are required to identify the molecular mechanism involved and the possible applications of the correlated genes in SKCM. The PPI network generated using the STRING online tool also identified nine CD38-interacting genes for correlation analysis in SKCM. The $\mathrm{GO}$ enrichment analysis revealed that the nine correlated genes (CD86, CD80, CD27, CD2, IL21R, IL2RB, ITGAL, CCL4 and TBX21) were involved in the regulation of $\mathrm{T}$ cell differentiation and lymphocyte activation. In particular, a previous study also identified CD2 as a correlated gene, indicating that CD2 might be an independent predictor of disease recurrence and OS in patients with primary cutaneous melanoma (27).

From the PPI network analysis, STRING and GeneMANIA identified 10- and 20-associated genes, respectively. Among the genes identified using STRING were genes from diverse functional groups, such as nicotinate-nucleotide adenylyltransferase activity (NMNAT1, NMNAT2 and NMNAT3), NAD ${ }^{+}$ nucleotidase and cyclic ADP-ribose generating [bone marrow stromal cell antigen-1 (BST1)] and pyrimidine metabolism (PNP, ENPP1 and ENPP3) genes. Several genes, such as NMNAT1, NMNAT2 and NMNAT3, have been previously identified to be involved in catalyzing $\mathrm{NAD}^{+}$synthesis in the nicotinate and nicotinamide metabolism pathways (31). The genes predicted using GeneMANIA were from various functional enrichments, such as response to virus (LCK, CD247, CD4, PIM2 and IFNAR2) and cellular response to alkaloid (RYR1, RYR2 and RYR3). BST1 was predicted as a CD38-associated protein by both databases. It has been reported that some SNPs in BST1 serve as predictors for Parkinson's disease (32). Moreover, a previous study revealed that a deletion involving CD38 and BST1 facilitates a fusion transcript in a patient with autism and asthma (33). GeneMANIA also revealed that ITGAL was associated with CD38, which is consistent with the correlation analysis from GEPIA2, and further supports the findings of a previous study that indicated that ITGAL might serve as a prognostic factor in patients with R0 resected Dukes stage B and C colorectal cancer (34). A previous study also investigated ITGAL as a prognostic factor in the survival of men with castration-resistant prostate cancer (CRPC), and SNPs in ITGAL may be associated with the risk of death in men with CRPC (35).

Nevertheless, further investigation is required to determine the potential mechanism and application value of the aforementioned CD38-associated genes in SKCM, including CD2, BST1 and ITGAL. In a previous study, photodynamic therapy was implemented for human melanoma $(5,6)$. Further studies developed related photodynamic therapy schemes and identified possible mechanisms $(36,37)$. Recent reports also demonstrate that targeting CD38 enhances antileukemic activity $(38,39)$. Therefore, CD38-targeted photodynamic therapy for SKCM requires further investigation.

In summary, the present study identified that CD38 was highly expressed in patients with SKCM and was associated with SKCM metastasis. Moreover, elevated expression of CD38 was associated with OS in patients with SKCM. The present study might aid the identification of the mechanism underlying SKCM progression and advance the development of antagonist CD38 strategies for patients with SKCM. However, a key limitation of the present study was that the data obtained from TCGA-SKCM lacked analysis of patients with CD38 positive and negative expression.

\section{Acknowledgements}

Not applicable.

\section{Funding}

The present study was supported by the National Natural Science Foundation of China (grant no. 31700736), China Scholarship Council (grant no. 201908420102), Hubei Medical Youth Tip-Top Talent, Leading Talent Program of Yangtze Talent Project and the College Students Innovative Entrepreneurial Training Program in Yangtze University (grant nos. 2018184 and 2019372).

\section{Availability of data and materials}

The dataset GDC TCGA Melanoma (SKCM) generated and/or analyzed during the present study are available in The Cancer Genome Atlas repository (https://portal.gdc.cancer.gov/).

\section{Authors' contributions}

XW and SH designed the study. XW and PW drafted the manuscript. XW, PW, LG, JW and SMASN performed the research and analyzed the data. XW, PW and LG revised the manuscript. All authors read and approved the final manuscript.

\section{Ethics approval and consent to participate}

The present study was approved and consented by the Ethics Committee of the Second Affiliated Hospital of Yangtze University.

\section{Patient consent for publication}

Not applicable.

\section{Competing interests}

The authors declare that they have no competing interests. 


\section{References}

1. Siegel RL, Miller KD and Jemal A: Cancer statistics, 2018. CA Cancer J Clin 68: 7-30, 2018.

2. Khunger A, Buchwald ZS, Lowe M, Khan MK, Delman KA and Tarhini AA: Neoadjuvant therapy of locally/regionally advanced melanoma. Ther Adv Med Oncol 11: 1758835919866959, 2019.

3. Krayem M, Sabbah M, Najem A, Wouters A, Lardon F, Simon S, Sales F, Journe F, Awada A, Ghanem GE and Van Gestel D: The benefit of reactivating p53 under MAPK inhibition on the efficacy of radiotherapy in melanoma. Cancers (Basel) 11: 1093, 2019.

4. Dummer R, Siano M, Hunger RE, Lindenblatt N, Braun R, Michielin O, Mihic-Probst D, von Moos R, Najafi Y, Guckenberger $\mathrm{M}$ and Arnold A: The updated Swiss guidelines 2016 for the treatment and follow-up of cutaneous melanoma. Swiss Med Wkly 146: w14279, 2016.

5. Lee EH, Lim SJ and Lee MK: Chitosan-coated liposomes to stabilize and enhance transdermal delivery of indocyanine green for photodynamic therapy of melanoma. Carbohydr Polym 224: 115143, 2019.

6. Shi L, Liu P, Wu J, Ma L, Zheng H, Antosh MP, Zhang H, Wang B, Chen $\mathrm{W}$ and Wang $\mathrm{X}$ : The effectiveness and safety of X-PDT for cutaneous squamous cell carcinoma and melanoma. Nanomedicine (Lond) 14: 2027-2043, 2019.

7. Cancer Genome Atlas Network: Genomic classification of cutaneous melanoma. Cell 161: 1681-1696, 2015.

8. Wang Q, Pan F, Li S, Huang R, Wang X, Wang S, Liao X, Li D and Zhang L: The prognostic value of the proteasome activator subunit gene family in skin cutaneous melanoma. J Cancer 10 2205-2219, 2019.

9. Wang Q, Wang X, Liang Q, Wang S, Xiwen L, Pan F, Chen H and Li D: Distinct prognostic value of mRNA expression of guanylate-binding protein genes in skin cutaneous melanoma. Oncol Lett 15: 7914-7922, 2018.

10. Xiong J, Bing Z and Guo S: Observed survival interval: A supplement to TCGA pan-cancer clinical data resource. Cancers (Basel) 11: 280, 2019.

11. Wang X, Song J, Wu Z, Fan B and Mode X: Dual roles of CD38 in autophagy. Yangtze Medicine 01: 8-19, 2017.

12. O'Steen S, Comstock ML, Orozco JJ, Hamlin DK, Wilbur DSS, Jones JC, Kenoyer A, Nartea ME, Lin Y, Miller BW, et al: The $\alpha$-Emitter astatine-211 targeted to CD38 can eradicate multiple myeloma in a disseminated disease model. Blood 134: 1247-1256, 2019.

13. Ben Baruch B, Blacher E, Mantsur E, Schwartz H, Vaknine H, Erez N and Stein R: Stromal CD38 regulates outgrowth of primary melanoma and generation of spontaneous metastasis. Oncotarget 9: 31797-31811, 2018.

14. Morandi F, Marimpietri D, Horenstein AL, Corrias MV and Malavasi F: Microvesicles expressing adenosinergic ectoenzymes and their potential role in modulating bone marrow infiltration by neuroblastoma cells. Oncoimmunology 8: e1574198, 2019.

15. Schutze K, Petry K, Hambach J, Schuster N, Fumey W, Schriewer L, Rockendorf J, Menzel S, Albrecht B, Haag F, et al CD38-Specific biparatopic heavy chain antibodies display potent complement-dependent cytotoxicity against multiple myeloma cells. Front Immunol 9: 2553, 2018.

16. Yu T, Qiao C, Lv M and Tang L: Novel anti-CD38 humanized mAb SG003 possessed enhanced cytotoxicity in lymphoma than Daratumumab via antibody-dependent cell-mediated cytotoxicity. BMC Biotechnol 19: 28, 2019.

17. Wang X, Peng X, Zhang X, Xu H, Lu C, Liu L, Song J and Zhang Y: The emerging roles of CIB1 in cancer. Cell Physiol Biochem 43: 1413-1424, 2017.

18. Morandi F, Morandi B, Horenstein AL, Chillemi A, Quarona V, Zaccarello G, Carrega P, Ferlazzo G, Mingari MC, Moretta L, et al: A non-canonical adenosinergic pathway led by CD38 in human melanoma cells induces suppression of T cell proliferation. Oncotarget 6: 25602-25618, 2015.

19. Chandrashekar DS, Bashel B, Balasubramanya SAH, Creighton CJ, Ponce-Rodriguez I, Chakravarthi BVSK and Varambally S: UALCAN: A portal for facilitating tumor subgroup gene expression and survival analyses. Neoplasia 19: 649-658, 2017.

20. Tang Z, Li C, Kang B, Gao G, Li C and Zhang Z: GEPIA: A web server for cancer and normal gene expression profiling and interactive analyses. Nucleic Acids Res 45(W1): W98-W102, 2017.
21. Szklarczyk D, Franceschini A, Wyder S, Forslund K, Heller D, Huerta-Cepas J, Simonovic M, Roth A, Santos A, Tsafou KP, et al: STRING v10: Protein-protein interaction networks, integrated over the tree of life. Nucleic Acids Res 43(Database Issue): D447-D452, 2015.

22. Franz M, Rodriguez H, Lopes C, Zuberi K, Montojo J, Bader GD and Morris Q: GeneMANIA update 2018. Nucleic Acids Res 46(W1): W60-W64, 2018.

23. Gao J, Aksoy BA, Dogrusoz U, Dresdner G, Gross B, Sumer SO, Sun Y, Jacobsen A, Sinha R, Larsson E, et al: Integrative analysis of complex cancer genomics and clinical profiles using the cBioPortal. Sci Signal 6: pl1, 2013.

24. Zhou Y, Zhou B, Pache L, Chang M, Khodabakhshi AH, Tanaseichuk O, Benner $\mathrm{C}$ and Chanda SK: Metascape provides a biologist-oriented resource for the analysis of systems-level datasets. Nat Commun 10: 1523, 2019.

25. Selitsky SR, Mose LE, Smith CC, Chai S, Hoadley KA, Dittmer DP, Moschos SJ, Parker JS and Vincent BG: Prognostic value of B cells in cutaneous melanoma. Genome Med 11: 36 , 2019.

26. Guan J, Gupta R and Filipp FV: Cancer systems biology of TCGA SKCM: Efficient detection of genomic drivers in melanoma. Sci Rep 5: 7857, 2015.

27. Harcharik S, Bernardo S, Moskalenko M, Pan M, Sivendran M, Bell H, Hall LD, Castillo-Martin M, Fox K, Cordon-Cardo C, et al: Defining the role of CD2 in disease progression and overall survival among patients with completely resected stage-II to -III cutaneous melanoma. J Am Acad Dermatol 70: 1036-1044, 2014

28. Hyams DM, Cook RW and Buzaid AC: Identification of risk in cutaneous melanoma patients: Prognostic and predictive markers. J Surg Oncol 119: 175-186, 2019.

29. van de Donk NWCJ, Richardson PG and Malavasi F: CD38 antibodies in multiple myeloma: Back to the future. Blood 131: 13-29, 2018.

30. Liu Q, Graeff R, Kriksunov IA, Lam CM, Lee HC and Hao Q: Conformational closure of the catalytic site of human CD38 induced by calcium. Biochemistry 47: 13966-13973, 2008.

31. Brazill JM, Li C, Zhu Y and Zhai RG: NMNAT: It's an NAD ${ }^{+}$ synthase. It's a chaperone. It's a neuroprotector. Curr Opin Genet Dev 44: 156-162, 2017.

32. Shen YT, Wang JW, Wang M, Zhi Y, Li JY, Yuan YS, Wang XX, Zhang H and Zhang KZ: BST1 rs4698412 allelic variant increases the risk of gait or balance deficits in patients with Parkinson's disease. CNS Neurosci Ther 25: 422-429, 2019.

33. Ceroni F, Sagar A, Simpson NH, Gawthrope AJ, Newbury DF, Pinto D, Francis SM, Tessman DC, Cook EH, Monaco AP, et al: A deletion involving CD38 and BST1 results in a fusion transcript in a patient with autism and asthma. Autism Res 7: 254-263, 2014.

34. Vendrell E, Ribas M, Valls J, Sole X, Grau M, Moreno V, Capella G and Peinado MA: Genomic and transcriptomic prognostic factors in R0 Dukes B and C colorectal cancer patients. Int J Oncol 30: 1099-1107, 2007.

35. Xie W, Stopsack KH, Drouin SJ, Fu H, Pomerantz MM, Mucci LA, Lee GM and Kantoff PW: Association of genetic variation of the six gene prognostic model for castration-resistant prostate cancer with survival. Prostate 79: 73-80, 2019.

36. Li Y, Xu Y, Peng X, Huang J, Yang M and Wang X: A novel photosensitizer $\mathrm{Znln}_{2} \mathrm{~S}_{4}$ mediated photodynamic therapy Induced-HepG2 cell apoptosis. Radiat Res 192: 422-430, 2019.

37. Wang X, He X, Hu S, Sun A and Lu C: Involvement of Bim in Photofrin-mediated photodynamically induced apoptosis. Cell Physiol Biochem 35: 1527-1536, 2015.

38. Roepcke S, Plock N, Yuan J, Fedyk ER, Lahu G, Zhao L and Smithson G: Pharmacokinetics and pharmacodynamics of the cytolytic anti-CD38 human monoclonal antibody TAK-079 in monkey-model assisted preparation for the first in human trial. Pharmacol Res Perspect 6: e00402, 2018.

39. Manna A, Aulakh S, Jani P, Ahmed S, Akhtar S, Coignet M, Heckman M, Meghji Z, Bhatia K, Sharma A, et al: Targeting CD38 enhances the antileukemic activity of ibrutinib in chronic lymphocytic leukemia. Clin Cancer Res 25: 3974-3985, 2019.

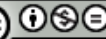

This work is licensed under a Creative Commons Attribution-NonCommercial-NoDerivatives 4.0 International (CC BY-NC-ND 4.0) License. 